\title{
Importance des problèmes de convection mixte et de stratification dans les réacteurs à neutrons rapides du type intégré
}

\author{
par H.J. Gollion \\ Chef du Service de Technologie \\ des Réacteurs à Sodium
}

\author{
et M. Sauvage \\ Chef de la Section de Développement \\ des Equipements des Réacteurs
}

Commissariat à l'Energie Atomique

Dépariement des Réacteurs à Neutrons Rapides - Service de Technologie des Réacteurs à Sodium

C.E.N., Cadarache

\section{Introduction}

Les réacteurs rapides refroidis au sodium, qu'ils soient déjà construits ou en cours d'études, relèvent toujours de l'un des deux concepts suivants:

- concept dit à circuits extérieurs;

- concept dit à circuits intégrés.

Les études et l'expérience acquises sur chacun de ces concepts ne permettent pas, aujourd'hui, de se prononcer totalement en faveur de l'un ou de l'autre de ces concepts, chacun ayant ses avantages et ses inconvénients.

Bien que le premier réacteur prototype français Rapsodie soit à circuits extérieurs, le C.E.A. s'est délibérément engagé pour les réacteurs suivants, Phénix et Super-Phénix, dans la voie des circuits intégrés, pour des raisons qu'il serait trop long d'exposer ici et qui n'ont rien à voir avec le sujet de cette conférence.

Les études engagées pour Phénix ont, rapidement, mis en évidence tout un ensemble de problèmes propres aux réacteurs intégrés qui, il faut bien l'avouer, avaient été quelque peu sous-estimés quand le changement de concept avait été proposé. Ce sont, essentiellement, les problèmes liés ati fait que l'on a un ensemble de structures contenant du sodium chaud plongées dans un sodium plus froid, ces structures étant de plus soumises au cours du temps à des fluctuations thermiques en relation avec les différents états de fonctionnement de la centrale. C'est l'étude de ces problèmes thermiques et hydrauliques que l'on englobe sous le nom de thermohydraulique.

Après avoir identifié, sur un schéma très simplifié, les zones affectées par ces problèmes, on présentera les différentes solutions qui ont été proposées au cours de l'étude et qui ont abouti au schéma retenu pour Phénix.

Pour terminer on dira quelques mots des problèmes thermohydrauliques de Super-Phénix et de la façon dont on pense les résoudre.

\section{Description \\ d'un réacteur rapide intégré refroidi au sodium}

Avant de décrire un réacteur du type intégré, on rappelle que les réacteurs rapides à sodium utilisent, entre le circuit (actif) extrayant la chaleur du cœur et le circuit de production de vapeur, un circuit intermédiaire - non actif également à sodium.

L'échange de chaleur entre ces deux circuits se fait au niveau d'un échangeur appelé échangeur intermédiaire.

Ce qui différencie les deux concepts, c'est que l'ensemble du circuit primaire, échangeur intermédiaire compris, peut être ou non contenu à l'intérieur d'une même cuve et immergé en sodium.

Un réacteur intégré se caractérise en effet par une grande cuve $(\varnothing 12 \mathrm{~m}$ sur Phénix) dite «cuve principale» (CP) contenant:

- L'ensemble des circuits primaires de la centrale, avec leurs principaux équipaments, les pompes primaires (PP) et les échangeurs intermédiaires (EI).

- Le cour constitué d'un ensomble d'éléments appelés assemblages, qui sont la source de chaleur de la centrale. La puissance thermique, ainsi produite, est extraite par une circulation forcée de sodium à l'intérieur des assemblages, le sodium étant distribué par un collecteur ou «sommier» (S) assurant également leur supportage; le sommier est relié aux pompes par des tuyauteries.

- Une cuve interne (CI) qui contient le sodium chaud (environ $560{ }^{\circ} \mathrm{C}$ ) sortant des assemblages. Plusieurs formes de cuves peuvent être proposées suivant la façon dont on alimente les échangeurs intermédiaires avec ce sodium chaud mais, pour la clarté de l'exposé, on retiendra celle qui a été adoptée pour Phénix et qui se caractérise par un large 
encorbellement, ou «redan», traversé par les pompes et échangeurs; le supportage est assuré par un platelage (P) suspendu à une virole (VC) reliée à la cuve principale.

La cuve interne, ainsi définie, sépare le sodium chaud sortant du cœur, du sodium froid (environ $400{ }^{\circ} \mathrm{C}$ ) sortant de la partie basse des échangeurs intermédiaires.

La zone comprise entre la cuve interne et la cuve principale est appelée «intercuve».

- Les protections neutroniques latérales (PNL) entoilrant le cœur qui reposent sur une structure appelée «fauxsommier » (FS).

- Un couvercle-cœur (CC), ensemble déffecteur du courant de sodium sortant des assemblages et qui assure également d'autres fonctions. Le couvercle est suspendu à un bouchon tournant (BT).

La cuve principale, dans Phénix, est refermée à sa partie supérieure par un «toit » relié à une structure porteuse, ou «dalle», à laquelle est également suspendue la cuve principale.

Enfin, on signale qu'un gaz neutre (argon), à une pression voisine de la pression atmosphérique, surmonte la surface libre du sodium.

On aboutit ainsi au schéma simplifié suivant, auquel nous nous rapporterons dans la suite (voir fig. 1).

\section{Zones \\ du réacteur posant des problèmes thermohydrauliques}

Devant un tel schéma, le mécanicien, responsable de la tenue des structures, ne peut manquer de se poser les questions suivantes:

- Quelle est la température exacte d'un point donné de la structure?

- Comment évolue-t-elle en fonction des différents régimes de température et de fonctionnement du réacteur?

- A quel gradient, telle partie de la structure est-elle soumise?

La réponse à ces questions est délicate, car on a affaire à des phénomènes complexes où entrent des variations de température dans l'espace et des variations de température dans le temps.

Les problèmes, liés aux variations spatiales de température, ont principalement pour cause la proximité d'un collecteur chaud avec des zones plus froides.

Des convections naturelles, favorisées par le faible nombre de Prandtl du sodium liquide, se développent alors le long des parois, transférant des puissances thermiques importantes d'une zone à une autre et créant de forts gradients de température dans les structures.

On notera que ces transferts internes conduisent à une dégradation du rendement de la centrale et à une diminution de la température du sodium chaud à l'entrée des échangeurs.

Les problèmes, liés aux variations temporelles de température, ont leur origine dans les différents régimes transitoires affectant la centrale.

Les régimes transitoires lents, tels que ceux rencontrés lors du démarrage ou de l'arrêt programmé de la centralic, présentent un caractère de moindre gravité que les régimes

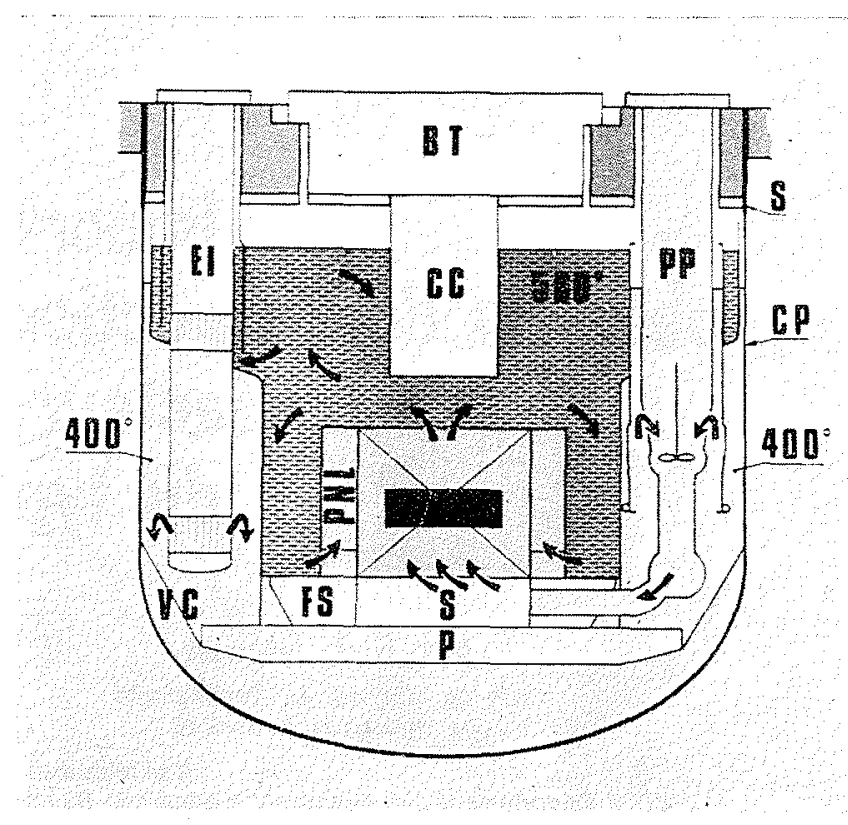

1/ Réacteur intégrê. Schéma hydraulique.

transitoires rapides, ou chocs thermiques, engendrés lors de l'arrêt de la centrale sur défaillance d'un élément.

Bien que le projeteur essaie de se prémunir contre ces transitoires rapides par divers artifices, il ne peut les éviter complètement. Les chocs les plus durs à considérer sont :

- un choc froid important (sur Phénix le choc est de $150^{\circ} \mathrm{C}$ d'amplitude avec un gradient de $5{ }^{\circ} \mathrm{C} / \mathrm{s}$ ) qui intéresse principalement la cuve interne; ce choc est consécutif à l'arrêt quasi-instantané de production de chaleur dans le cœur;

- un choc chaud également important (sur Phénix le choc pris en compte est de $128^{\circ} \mathrm{C}$ d'amplitude, avec un gradient maximum de $5{ }^{\circ} \mathrm{C} / \mathrm{s}$ ) qui prend naissance à la suite d'un arrêt brutal de la circulation dans un circuit secondaire et qui affecte principalement l'intercuve.

On notera ici un des avantages du concept intégré qui présente des masses importantes de sodium dans le collecteur chaud et le collecteur froid.

Ce sodium, par son mélange avec le sodium sortant du cour ou des échangeurs, amortit considérablement les chocs thermiques, ce qui simplifie la protection à mettre en place sur les structures environnantes.

Si on revient au schéma présenté précédemment, on peut isoler un certain nombre de zones, dont le calcul est difficile, du fait des problèmes de thermohydraulique; il s'agit principalement :

$1^{\circ}$ De la virole support du platelage et du platelage proprement dit dont la stabilité doit être garantie en toutes circonstances.

2" Du faux sommier supportant les éléments de protection entourant le cœur - qui lui aussi doit rester stable dans le temps - Les éléments de protection étant refroidis par convection naturelle, il faut d'autre part être sûr du champ de température sur toute la hauteur des éléments.

$3^{\circ}$ Du redan de la cuve interne, dont le raccordement à la partie cylindrique de la cuve doit être particulièrement 


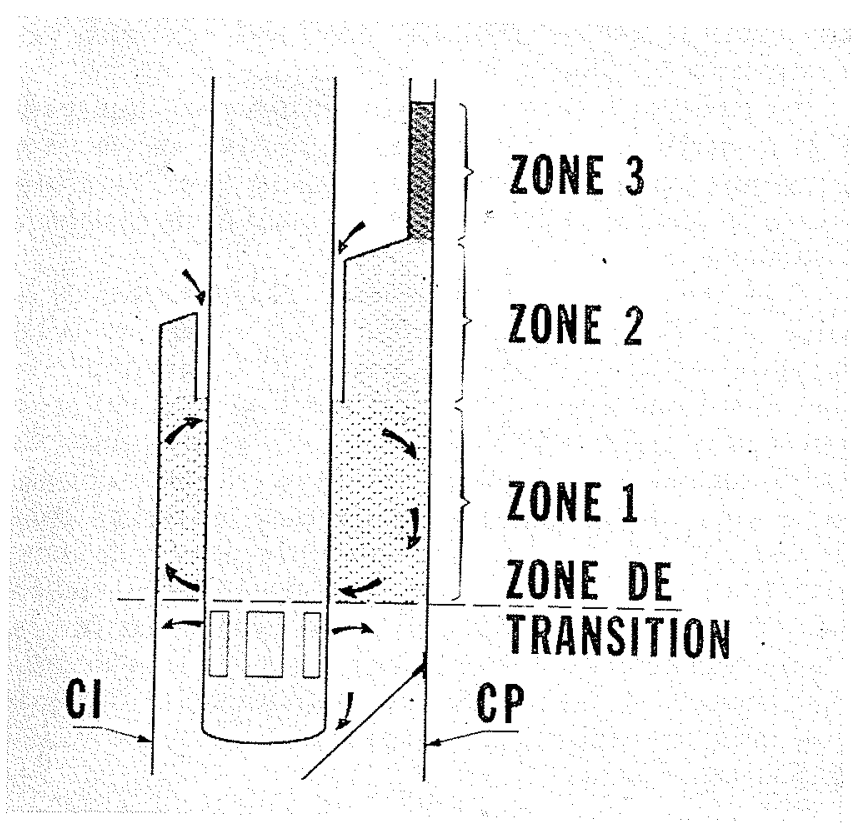

2/ Thermique de l'intercuve.

soigné. Le redan est sensible à la fois aux gradients longitudinaux dans la partie courante et aux gradients transversaux.

$4^{\circ}$ La cuve interne dans sa partie cylindrique inférieure, qui est sensible principalement aux gradients transversaux.

Des quatre zones ainsi identifiées, celles qui posent le plus de problèmes sont, sans aucun doute, la cuve interne et son redan. Les températures, prises par cette structure, dépendent à la fois des conditions thermiques régnant dans le sodium chaud qu'elle contient et des conditions thermiques dans l'intercuve.

Cette cuve et son redan sont soumis, sur leur face interne, aux chocs thermiques froids prenant naissance dans le cœur. $\mathrm{Si}$ on veut les amortir, il faut assurer, dans le collecteur chaud, un brassage correct du sodium par des mouvements de convection forcée.

Sur sa face externe, la cuve interne et son redan sont soumis aux conditions thermiques régnant normalement en régime établi et à des transitoires de températures consécutifs, par exemple, à un incident sur un circuit secondaire (choc chaud).

Une analyse qualitative de la thermique régnant dans l'intercuve en régime établi permet de délimiter trois zones soumises à des températures bien différentes (voir fig. 2).

Le sodium de l'intercuve est en effet:

- rechauffé par les fuites thermiques à travers la cuve interne;

- rechauffé par le débit de fuite du sodium chaud s'écoulant au niveau des traversées du redan par les échangeurs (dans le cas de traversées non étanches);

- refroidi par les fuites thermiques vers les viroles des pompes d'échangeurs, vers l'extérieur de la cuve principale, ainsi que vers le sodium froid sortant des échangeurs.

$\mathrm{Du}$ point de vue écoulement, on peut présumer :

- Que des boucles de convection naturelles vont se développer. Le sodium va s'écouler du haut en bas, en se refroidissant le long des viroles froides de la cuve principale, et de bas en haut, en s'échauffant le long de la cuve interne.

Ceci se produit approximativement dans la zone 1 de la figure 2.

La partie inférieure de cette zone, dans laquelle s'établissent des courants de circulation, est «grignotée » en permanence par les courants de circulation forcée qui règnent entre pompes et échangeur dans la partie basse de l'intercuve. Il y a établissement d'une zone à fort gradient de température (ou zone de transition) dont la cote en altitude est fonction du régime des pompes.

- Que les fuites de sodium chaud de la cuve interne vont avoir tendance à s'accumuler en partie haute de la zone 2.

- Que la zone 3 est pratiquement isotherme car peu renouvelée.

\section{Différentes solutions envisagées pour Phénix}

Les efforts ont surtout porté sur la thermohydraulique de l'intercuve, car il était certain que l'existence de cette zone de transition était fortement préjudiciable au comportement des structures dans la zone où elle s'établissait.

La stabilité de la virole de supportage du platelage, devant les différents régimes de température de la centrale, a été acquise assez simplement en reportant cette structure dans une zone thermiquement stable (aux alentours de $400{ }^{\circ} \mathrm{C}$ ) peu affectée par les courants de sodium circulant dans l'intercuve, donc protégée contre d'éventuels chocs thermiques.

La stabilité du faux-sommier et le refroidissement en convection naturelle des éléments de protection neutronique latérale a été obtenue au moyen d'un déflecteur fixé sur la paroi de la cuve interne dont le rôle essentiel était d'éviter aux courants de sodium chaud, déviés par le couvercle cœur et venant frapper la paroi interne de la cuve, de se diriger vers le faux-sommier et d'aller ainsi le rechauffer.

Des courants de convection naturelle s'établissent ainsi de bas en haut autour des éléments de protection (zone chaude) et de haut en bas le long de la paroi de la cuve interne (zone froide).

En ce qui concerne la thermohydraulique de l'intercuve, on a envisagé les solutions suivantes:

a) La première idée qui vient à l'esprit, est d'assurer un brassage efficace de l'intercuve afin d'empêcher la zone de transition de se créer. Pour cela on peut :

- soit aspirer, avec les pompes près de la surface, le sodium rejeté vers le bas par les échangeurs (schéma a, fig. 3); ce système a pour inconvénient de produire un entraînement de gaz et de nécessiter un niveau de sodium stable;

- soit aspirer avec les pompes, vers le fond, le sodium rejeté vers le haut par les échangeurs au moyen d'un déflecteur (schéma b, fig. 3); les inconvénients de ce système sont d'introduire des pertes thermiques non négligeables à travers le redan et de soumettre le redan à un gradient thermique important, en particulier pendant les transitoires de température, ce qui rend sa tenue mécanique trop délicate. 
b) Ces solutions ayant été abandonnées devant les inconvénients qu'elles présentaient, on s'est orienté vers la mise en place d'écrans thermiques latéraux le long des cuves interne et principale, ce qui les protège des effets thermiques de la zone de transition (schéma c, fig. 3).

Dans ce schéma, où la température du redan est homogène, les difficultés sont venues de l'impossibilité de localiser exactement le niveau de la zone de transition et d'apprécier la valeur du gradient qu'elle crée. Le problème s'est compliqué par les incertitudes qui entouraient la valeur des fuites thermiques aux parois et la valeur du débit de fuite aux échangeurs.

On se heurte également à une difficulté supplémentaire au niveau des écrans verticaux : on doit, en effet, assurer entre chacun d'eux, un écartement faible, de façon à cc qu'il ne puisse pas se créer, dans l'intervalle qui les sépare. des courants de convection naturelle ou forcée qui les rendraient totalement inefficaces.

\section{Schéma retenu pour l'intercuve de Phénix}

Le schéma qui a été finalement retenu pour l'intercuve de Phénix, diffère notablement des solutions précédentes pour une raison fort éloignée de la thermohydraulique et qui a été imposée aux projeteurs en cours d'étude.

Il a en effet été demandé, suite à des considérations métallügiques, de limiter la température maximale de la cuve principale à une valeur inférieure à $500{ }^{\circ} \mathrm{C}$. Ceci imposait de refroidir la cuve principale par un débit de sodium dérivé, ce qui a été obtenu par la mise en place d'une cuve

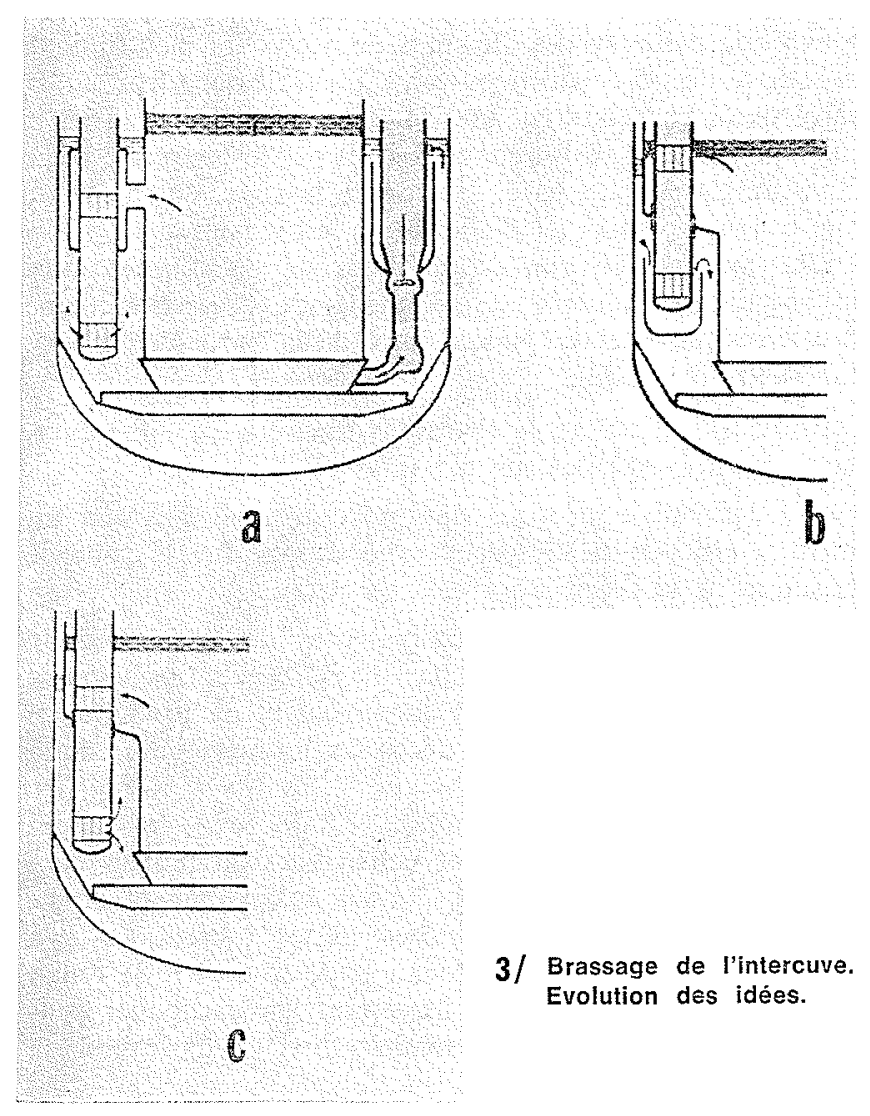

canalisant le sodium provenant des fuites aux pieds des assemblages, le long de la cuve principale (cf. fig. 4).

Parallèlement à cette importante modification, qui résolvait le problème des contraintes thermiques sur la cuve principale, on a supprimé complètement toute fuite aux traversées d'échangeur par la mise en place d'une étanchéité pneumatique.

Dans cette solution, le sodium de refroidissement de la cuve principale (débit de lordre de 7 à $8 \%$ du débit nominal, température au point haut : $420^{\circ} \mathrm{C}$ ) a été réintroduit dans l'intercuve à un niveau assez bas, pour être assez rapidement aspiré par les pompes. La paroi interne du baffie, canalisant ce sodium, constitue un échangeur efficace qui a pour effet d'abaisser la température du sodium dans la zone au-dessus du rejet. La température, dans cette zone, est à peu près homogène du fait des courants de convection naturelle qui s'y établissent et elle se situe à un niveau intermédiaire entre la température du sodium chaud et celle du sodium froid. En fait, la zone de transition ne disparaît pas, mais son gradient de température s'en trouve considérablement atténué.

Par contre, avec ce schéma, est apparu un nouveau problème, lui aussi très délicat à résoudre. Le circuit de refroidissement de la cuve principale présente dans sa partie haute, au niveau de retournement du sodium, une zone morte importante à laquelle il est difficile d'échapper à moins d'accepter un dénoyage du baffle de retournement à certains régimes thermiques de la centrale (schéma a, fig. 5).

La température, dans cette zone, gouverne alors l'établissiment de gradients longitudinaux dans la partie de la cuve se raccordant au toit. Ainsi, au cours des différents régimes thermiques (transitoires lents) du réacteur, les températures dans cette zone sont-elles différentes de celles prises par le toit, du fait de son inertie.

Sur Phénix, cette difficulté a été tournée au moyen d'un artifice consistant à tapisser la zone en question de résistances électriques, chauffant la structure à la valeur désirée.

\section{Solutions envisagées pour Super-Phénix}

Les problèmes de thermohydraulique, posés pour un réacteur de grande taille tel que Super-Phénix, où la cuve principale atteint un diamètre de l'ordre de $21 \mathrm{~m}$, sont de même nature que ceux rencontrés sur Phénix. L'organisation interne des structures étant similaire, les solutions retenues pour Phénix ont été reconduites dans la plupart des cas.

Les seules différences importantes qu'il y a lieu de signaler concernent:

- la forme de la cuve interne et la position du redan qui ont changé;

- la configuration de la zone de retournement, pour laquelle on est toujours à la recherche de solutions satisfaisantes.

\section{Cuye interne ef redan (fig. 6)}

Pour des raisons propres à la manutention du combustible, et également pour une question de résistance mécanique du redan, le diamètre de la cuve interne a été notablement 


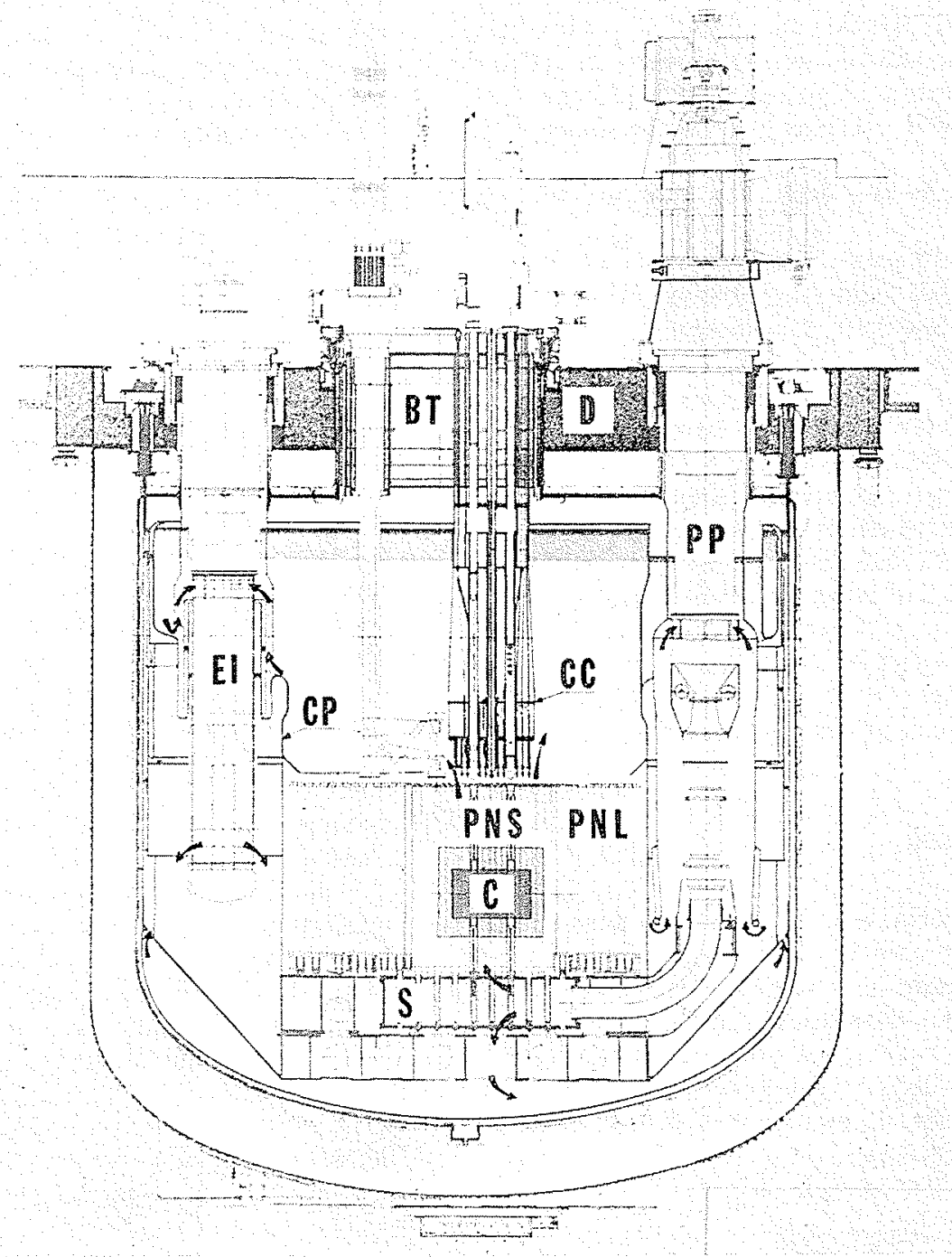

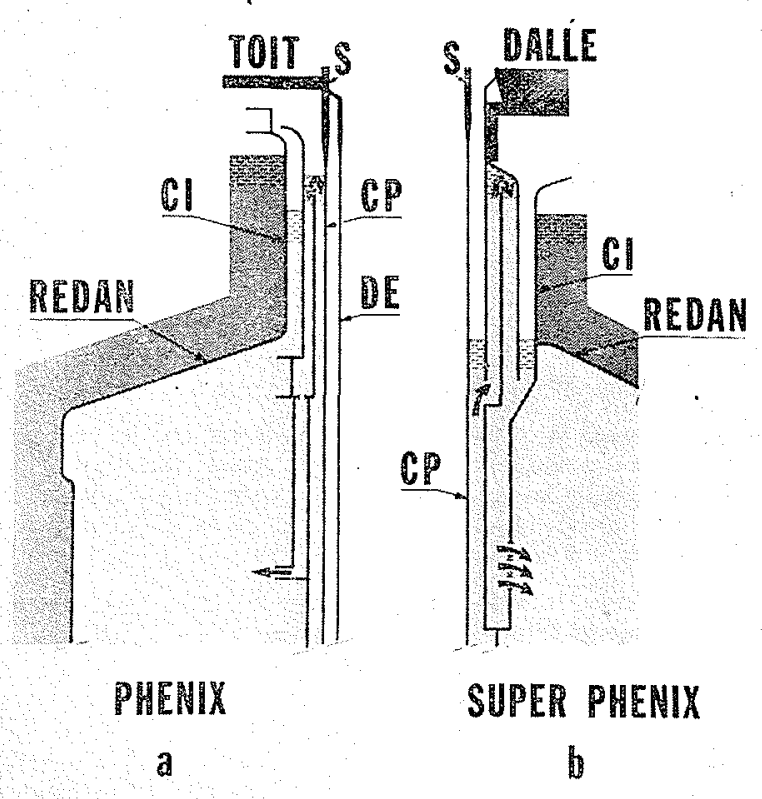

5/ Zone de retournement.

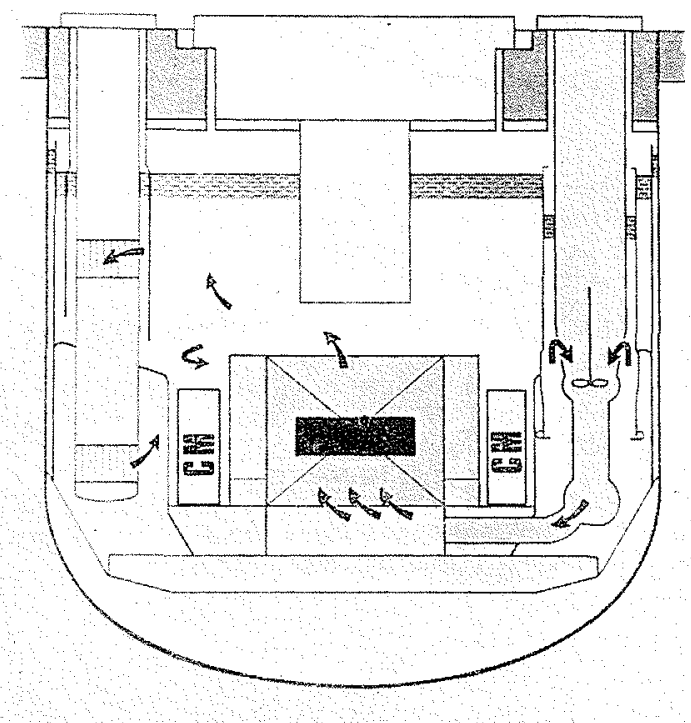

6/ Super-Phénix. Coupe bloc réacteur. 
augmenté, de telle sorte qu'on a, entre la cuve interne et la protection neutronique latérale, un important espace libre. Simultanément à cette modification, le niveau du redan a été abaissé relativement à la position qu'il occupait dans Phénix, c'est-à-dire qu'il est plus proche des fenêtres de sortie des échangeurs.

Les conséquences de ces deux modifications sont les suivantes:

L'abaissement du niveau du redan évite au jet, déflecté par le couvercle cour, de venir frapper directement la paroi de la cuve interne. Les viroles d'aspiration des échangeurs ayant été corrélativement rallongées, le jet de sodium, sortant du cœur, vient heurter ces viroles ainsi que celles entourant les pompes, favorisant ainsi un meilleur brassage du sodium dans le collecteur chaud.

$\mathrm{Si}$ on veut, d'autre part, maintenir le faux-sommier à une température suffisamment basse et garantir une circulation de sodium par convection naturelle dans la protection neutronique latérale, il faut impérativement combler l'espace libre entre la protection et la cuve interne, sous peine de voir s'y établir des courants de circulation de sodium chaud en provenance du cœur.

On étudie actuellement comment y parvenir.

\section{Zone de retournement}

On a vu que, sur Phénix, le sodium stagnant dans la zone de retournement avait créé des problèmes mécaniques et thermiques dans la partie hors sodium des structures. On retrouve un tel problème dans Super-Phénix et, pour éviter la formation de cette zone morte au retournement, on a proposé une solution utilisant un siphon qui a le lourd inconvénient de multiplier le nombre des structures métalliques (voir fig. 5, schéma b).

Comme dans Phénix, le sodium de refroidissement de la cuve est ensuite canalisé vers l'intercuve et réinjecté à un niveau tel qu'il crée une zone tampon, protégeant le redan des transitoires thermiques en provenance des échangeurs intermédiaires.

Fonctionnellement, ce genre de solution est tout à fait satisfaisant. Par contre, les structures à mettre en place deviennent compliquées at elles coûtent cher. Aussi, recherche-t-on, actuellement, d'autres solutions plus simples, sur lesquelles on ne peut encore se prononcer, l'étude n'étant pas terminée.

\section{Conclusion}

Comme on vient de le voir, les problèmes de thermohydraulique, qui se sont posés aux concepteurs des réacteurs intégrés du type Phénix, ont pu trouver des solutions satisfaisantes grâce à un certain nombre de dispositions, plus ou moins complexes, qui ont essentiellement pour objet d'établir des courants de circulation privilégiés le long des structures et d'imposer ainsi la thermique de ces parties.

C'est dire toute l'importance qui est attachée, en premier lieu, à la très bonne connaissance de l'hydraulique du système, laquelle n'a été acquise qu'à la suite d'essais à l'eau très complets sur des maquettes à échelle réduite.

Comme on le voit avec Super-Phénix, chaque nouvelle génération de réacteurs pose ses propres problèmes, qui viennent s'ajouter à ceux soulevés par le projeteur quand il essaye de simplifier le concept. Cette simplification du dessin ne pourra être totalement obtenue que lorsqu'on connaîtra mieux les lois qui régissent l'établissement des mouvements de convection du sodium le long des parois des structures et que l'on saura mieux apprécier les effets des jets sortant des échangeurs dans les espaces fermés, tels que l'intercuve des réacteurs du type intégré.

\section{Discussion}

M. le Président remercie M. Sauvage pour son exposé qui montre clairement comment se posent les différents problèmes qui seront évoqués dans les autres exposés figurant à l'ordre du jour de la séance. Il ouvre ensuite la discussion.

Sur tine question de M. Geffroy, M. Sauvage apporte les précisions suivantes :

En cas de «perte totale» du réseau, si les pompes ne sont pas alimentées, on montre qu'il s'établit une convection naturelle dans le «cœur», de même sens que la circulation forcée qui évacue la puissance résiduelle dissipée par le cour.

Si l'on ne fait rien, la convection naturelle s'établit d'elle-même mais il peut en résulter des températures dangereuses, non pas pour la sûreté mais pour la «santé du cour».
M. Mallet pose la question «historique » ci-après :

Si, au moment du choix entre la version «intégrée» et la version «en circuit», on avait acquis la certitude que pour des raisons de métallurgie, la température de la cuve ne devait pas dépasser $500^{\circ} \mathrm{C}$, la solution retenue n'aurait-clle pas été différente?

Personnellement, M. Sauvage ne le pense pas, car les réacteurs à circuits intégrés présentent des avantages appréciables au point de vue si important de la sûreté. D'ailleurs, ajoute M. LaLlement, il est possible que pour des raisons de métallurgie, on aurait dû aussi refroidir un réacteur «à boucles».

M. le Président clôt la discussion et donne la parole à M. Malmerbe pour l'exposé de la communication suivante. 